\title{
Subarachnoid hemorrhage: a rare presentation of cerebral venous thrombosis - a case report
}

\begin{abstract}
Diagnosis of cerebral sinus venous thrombosis (CSVT) requires high index of suspicion owing to the varied spectrum of its clinical manifestations. Its diagnosis may be more elusive if the patient presents with subarachnoid hemorrhage (SAH). We report a case of CSVT that presented with SAH without underlying parenchymal involvement and will elaborate the importance of the early diagnosis of CSVT. Twenty-three years old woman presented with thunderclap headache and neuroimaging initially suggested SAH with no associated parenchymal bleeding. SAH involved the sulci of the convexity and spared the basal cisterns. Angiography revealed occlusion of intracranial venous sinuses without revealing any other cause of SAH. She showed marked improvement with anticoagulant therapy. In our observation, isolated CSVT can present solely as unilateral, localized SAH at the convexity without underlying parenchymal involvement. Because other causes of nontraumatic SAH do not frequently present in this manner, identification of this unusual imaging appearance should prompt strong consideration of the diagnosis of cortical sinus venous thrombosis.
\end{abstract}

Keywords: cerebral sinus venous thrombosis, subarachnoid hemorrhage, thunderclap headache
Volume 6 Issue I - 2017

\author{
Lakshya Jyoti Basumatary,' Ashish Sardhara, ${ }^{2}$ \\ Nitin Nyharkar, ${ }^{2}$ Mrinal Singha, ${ }^{3}$ Sandip \\ Chabukswar ${ }^{2}$ \\ 'Visiting Neurologist, Down town Hospital, India \\ ${ }^{2}$ DNB Medicine, Down town Hospital, Guwahati, India \\ ${ }^{3}$ Consultant Radiologist, Down town Hospital, Guwahati, India
}

Correspondence: Dr. Lakshya Jyoti Basumatary, Visiting Neurologist, down town Hospital, Guwahati, India, Pin 7816, Tel +91 9954480668, Email drbasumatary@gmail.com

Received: October 18, 2016 | Published: January 23, 2017

\section{Introduction}

Cerebral sinus venous thrombosis (CSVT) can present with a wide spectrum of symptoms and signs. ${ }^{1-3}$ Headache is the commonest presentation which is usually diffuse \& gradual in onset. A minority of patients may present with thunderclap headache, suggestive of subarachnoid hemorrhage, and a migrainous type of headache has also been described. ${ }^{4}$ Other presentations include seizures, papilloedema, focal deficits and impairment of consciousness. We are reporting a case of CSVT presented with subarachnoid hemorrhage.

\section{Case history}

A 23 year old female with history of migraine presented to emergency department with 3 days history of severe holocranial headache \& recurrent epiosodes of vomiting. She had history of episodic hemicranial headache for last 2 years and was diagnosed as migraine without aura. She was taking ergotamine for last 1 year as an over the counter medicine from pharmacy on and off. She is a regular smoker \& ethanolic. She also admitted occasional indulgence in cannabis and cocaine.

On examination she was conscious, oriented $\&$ afebrile. Her pulse was 80 beats per minute, regular \& blood pressure was $120 / 80 \mathrm{~mm}$ $\mathrm{Hg}$. Eyes showed conjunctival congestion \& non pulsatile soft tissue swelling over right eye lid. There were no cranial nerve palsies or focal neurological deficit. Meningeal signs were positive. Fundoscopy showed bilateral papilloedema.

Laboratory tests revealed normal erythrocyte sedimentation rate, white blood cell count and thrombocyte count. Non contrast CT scan brain showed cortical subarachnoid haemorrhage and mild gyral edema in right parietal lobe without any blood in the cisterns (Figure 1A). CT- contrast enhanced image indicates the filling defect caused by the thrombus in the superior sagittal sinus (Figure 1B). MRI brain with MR venogram confirmed thrombosis of superior sagittal sinus \& non visualization of straight sinus. Immunological screening tests including those for anti-dsDNA, anti-neutrophilic cytoplasmic antibodies, antinuclear antibodies, anticardiolipin were all negative.
Coagulation testing including levels of protein $\mathrm{C}$ and $\mathrm{S}$, prothrombin time, and activated partial thromboplastin time, antithrombin III and fibrinogen were all within normal limits. She was managed conservatively. She was started on IV low molecular weight heparin followed by oral anticoagulant.
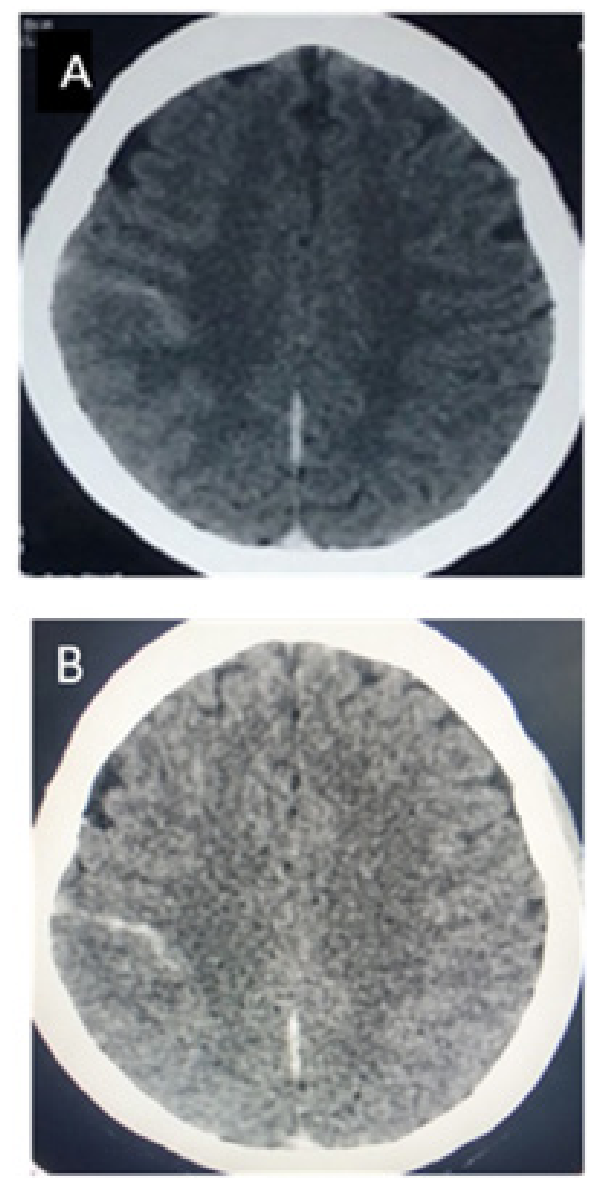

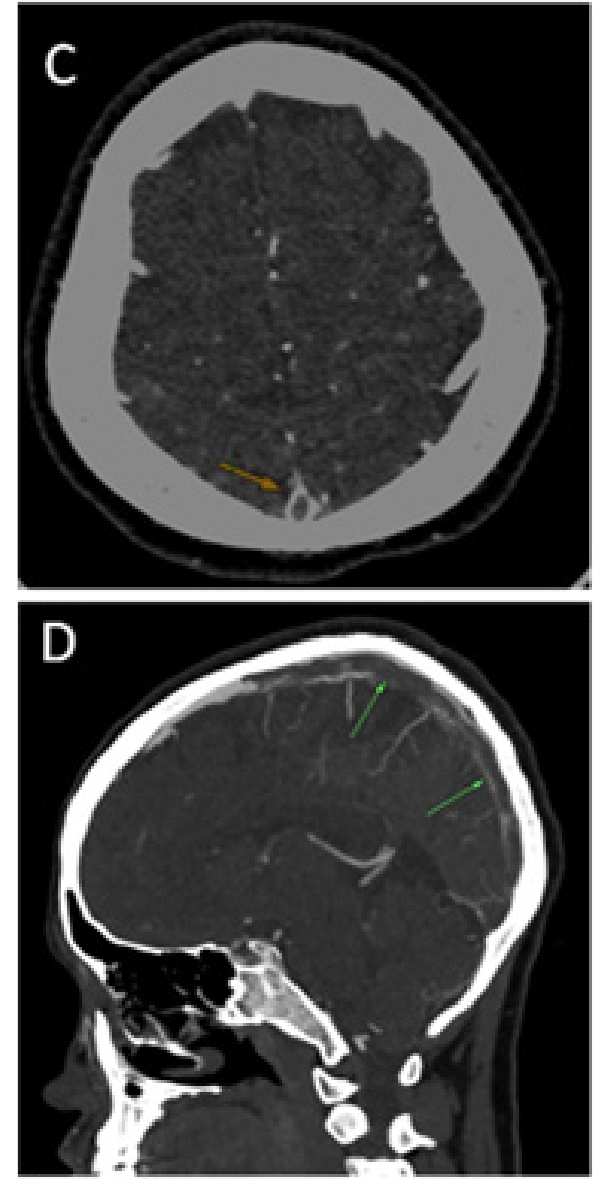

Figure I (A) Non-contrast \& contrast brain computed tomography (CT) showed hyperdensity over the right parietal sulci suggestive of in acute subarachnoid hemorrhage. (C) Brain CT with contrast revealed an empty delta sign in the superior sagittal sinus thrombus (arrow). Reconstructed sagittal CT- contrast enhanced image indicates the filling defect caused by the thrombus in the superior sagittal sinus.
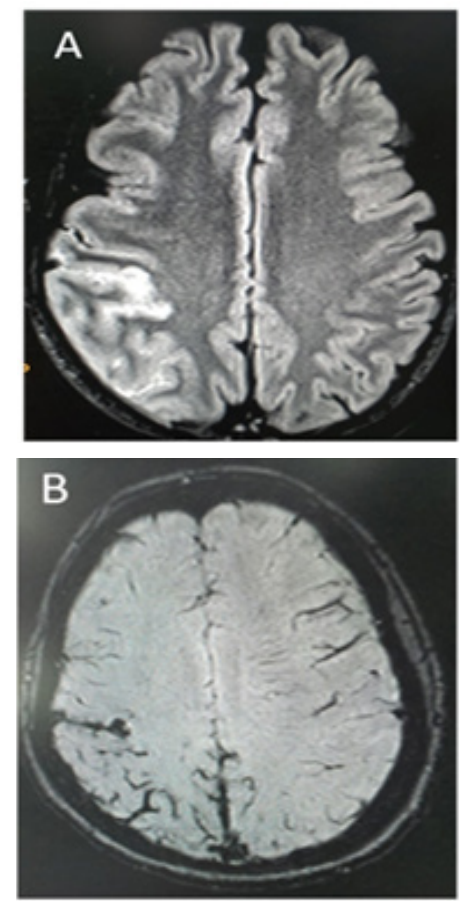

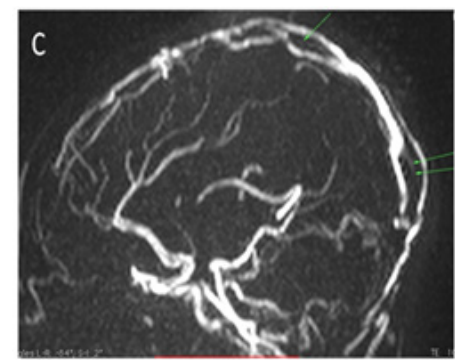

Figure 2 (A) MRI TI FLAIR showing hyperintense signal in right parietal sulci suggestive of subarachnoid hemorrhage. (B) MRI T2 weighted image hypointense signal in the posterior sagittal sinus with loss normal flow signal and in the parietal sulci. (C) $3 \mathrm{~d}$ Phase contrast MR venography images shows filling defects in the superior sagittal sinus and non visualization of straight sinus.

\section{Discussion}

The most common cause of nontraumatic SAH is rupture of an intracranial aneurysm $(85 \%) .{ }^{5}$ Less common causes are blood dyscrasias, CSVT(both dural and cortical), ${ }^{6,7}$ reversible cerebral vasoconstriction syndrome(RCVS), ${ }^{8}$ posterior reversible encephalopathy syndrome 9 , cerebral amyloid angiopathy(CAA), primary, metastatic or meningeal neoplasms, vasculitis, moyamoya disease or syndrome, ${ }^{10}$ drug abuse, cerebral venous occlusive disease, bacterial meningitis, spinal cord vascular malformations and spinal cord tumors, arterial dissection, dural arteriovenous fistula and pituitary apoplexy. ${ }^{5-12} \mathrm{SAH}$ is increasingly recognized as a presentation, both for cortical and dural sinus thrombosis. ${ }^{6,7}$ The diagnosis of CSVT becomes difficult when it presents with acute SAH. ${ }^{6}$ The distribution of a subarachnoid hemorrhage from a superior sagittal sinus thrombosis is usually different from that of one with an arterial origin and has a characteristic pattern. The hemorrhage is usually limited to the sulci of the cerebral convexity, sparing the basal cisterns. ${ }^{6}$ The exact mechanism of SAH associated with CSVT is not known but may result from venous hypertension and subsequent rupture of dilated thin-walled subarachnoid cortical veins. ${ }^{6}$

In our case patient had risk factors for CSVT, alcohol and cocaine abuse. Initial CT scan (non-contrast and contrast) showed cortical $\mathrm{SAH}$ in right parietal lobe without blood in basal cistern probably due to thrombosis of superior sagittal sinus. CT angiography and MR imaging done to confirm the diagnosis. Magnetic resonance imaging (MRI) and magnetic resonance venography (MRV) are the best test to diagnose and follow-up of CSVT.

In nontraumatic cortical SAH sparing of the basal cisterns an important radiological clue pointing towards CSVT, so clinician should promptly get imaging of both intracranial arteries and venous sinuses because the management of venous SAH secondary to CSVT is also quite different from that of arterial SAH. The usual treatment of sinus thrombosis is anticoagulation or local thrombolysis. Systemic anticoagulation is the first line treatment for CSVT because of its efficacy, safety, and feasibility. ${ }^{13}$ Our case showed significant improvement after treatment with anticoagulation.

\section{Conclusion}

SAH can be rare initial presentation of CSVT. Important radiological clue is the presence of subarachnoid blood in the cerebral convexities sparing the basal cisterns even in the absence of parenchymal lesions. The treatment with anticoagulation improves the outcome. 


\section{Acknowledgments}

None.

\section{Conflicts of interest}

None.

\section{Funding}

None.

\section{References}

1. Stam J. Thrombosis of the cerebral veins and sinuses. $N$ Engl J Med. 2005;352(17):1791-1798.

2. Bousser MG, Ferro JM. Cerebral venous thrombosis: an update. Lancet Neurol. 2007;6(2):162-170.

3. de Bruijn SF, Stam J, Kappelle LJ. Thunderclap headache as first symptom of cerebral venous sinus thrombosis: CVST Study Group. Lancet. 1996;348(9042):1623-1625.

4. Cumurciuc R, Crassard I, Sarov M, et al. Headache as the only neurological sign of cerebral venous thrombosis: a series of 17 cases. $J$ Neurol Neurosurg Psychiatry. 2005;76(8):1084-1087.

5. Van Gijn J, Rinkel GJ. Subarachnoid haemorrhage: diagnosis, causes and management. Brain. 2001;124(Pt 2):249-278.
6. Oppenheim C, Domigo V, Gauvrit JY, et al. Subarachnoid hemorrhage as the initial presentation of dural sinus thrombosis. AJNR Am J Neuroradiol. 2005;26:614-617.

7. Benabu Y, Mark L, Daniel S, et al. Cerebral venous thrombosis presenting with subarachnoid hemorrhage: case report and review. Am J Emerg Med. 2009;27(1):96-106.

8. Ducros A, Boukobza M, Porcher R, et al. The clinical and radiological spectrum of reversible cerebral vasoconstriction syndrome: a prospective series of 67 patients. Brain. 2007;130(pt 12):3091-3101.

9. Shah AK. Non-aneurysmal primary subarachnoid hemorrhage in pregnancyinduced hypertension and eclampsia. Neurology. 2003;61(1):117-120.

10. Osanai T, Kuroda S, Nakayama N, et al. Moyamoya disease presenting with subarachnoid hemorrhage localized over the frontal cortex: case report. Surg Neurol. 2008;69(2):197-200.

11. Bousser MG, Chiras J, Bories J, et al. Cerebral venous thrombosis -- a review of 38 cases. Stroke. 1985;16(2):199-213.

12. Warlow CP, Dennis MS, van Gijn J. Stroke: A Practical Guide to Management. Oxford: Blackwell Science, USA. 2001. p.376-413.

13. Rodallec MH, Krainik A, Feydy A, et al. Cerebral venous thrombosis and multidetector CT angiography: tips antd tricks. Radiographics. 2006;26 (suppl 1):S5-S18. 